\title{
A systems view of Drosophila segmentation Mike Levine
}

Address: Department of Molecular and Cell Biology, Division of Genetics, Genomics and Development, Center for Integrative Genomics, University of California, Berkeley, CA 94720, USA. Email: mlevine@berkeley.edu

Published: II February 2008

Genome Biology 2008, 9:207 (doi:10.1 I86/gb-2008-9-2-207)

The electronic version of this article is the complete one and can be

found online at http://genomebiology.com/2008/9/2/207

(C) 2008 BioMed Central Ltd

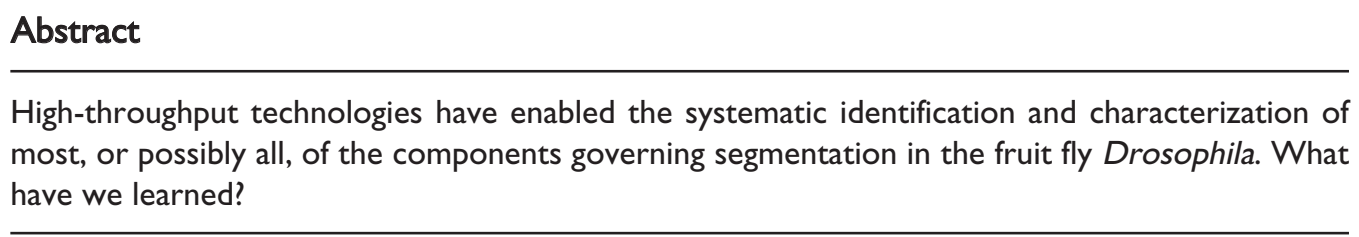

One of the most intensively studied processes in animal development is the division of the embryonic Drosophila epidermis (ectoderm) into visible segments, a process that lays the foundations for the segmented structure of the adult insect. Segmentation is governed by a program of sequential gene expression that is one of the best-defined genetic cascades in animal development [1]. It is put into motion by three maternal gene regulatory proteins - Bicoid, Hunchback and Caudal - which specify an initial 'pre-segmentation' pattern along the anterior-posterior axis, while the anterior and posterior ends of the body are specified independently by the localized activation of the maternal receptor tyrosine kinase Torso. The principal target genes of these maternal factors in the embryo's genome are known as gap genes, as their lack leads to gaps in the body pattern. The gap genes, such as Krüppel, Knirps and Giant, encode sequence-specific transcriptional repressors. The interplay of the maternal factors and the gap repressors constitutes one of the leading paradigms for the combinatorial control of gene expression in development. These regulatory factors bind to the enhancers of the segmentation genes to produce precisely positioned on/off repeating transverse stripes of expression for each gene, foreshadowing the subdivision of the embryo into a repeating series of body segments. The segmentation genes typically have highly complex enhancers, with multiple binding sites for each gene regulatory protein.

Detailed descriptions of individual parts of the Drosophila segmentation process have been available for many years, but Segal and colleagues [2] have recently taken a systems approach to segmentation by developing a comprehensive quantitative thermodynamics-based model in an attempt to describe the regulation of segmentation-gene expression in the early embryo. This analysis was made possible by two recent advances in understanding Drosophila segmentation. First, computational and experimental methods have identified a total of 44 different enhancers that respond to distinct combinations of maternal and gap transcription factors to direct precisely localized bands and stripes of gap and segmentation gene expression in the early Drosophila embryo [3-6]. Second, quantitative high-resolution imaging of the distribution profiles of each maternal and gap protein has provided precise information about the relative concentrations of these critical regulators in each nucleus of the embryo at key points during the progressive refinement of stripe formation $[7,8]$. Each enhancer contains a specific constellation of binding sites for maternal and gap proteins, and within each nucleus there is a particular combination of transcriptional activators and repressors that can bind to these sites.

Using this information, Segal et al. devised a two-component model to predict the expression, or output, mediated by each of the 44 known gap gene and segmentation gene enhancers [2]. The model incorporates some of the features used in previous site-occupancy models - such models predict the occupancy of each binding site by its corresponding transcription factor for a given gene and position in the embryo (for example, see [9-11]). The first component of the Segal model is a Boltzmann weight function (which will predict the probability that a binding site is occupied at a given concentration of transcription factor), which is computed for every sequence match to a known binding motif within a given enhancer. The second component of the model is a function that defines the transcriptional output, which depends on 
the ratio of predicted activator and repressor binding sites occupied. Higher occupancy of the activator sites is predicted to produce expression at a particular location within the embryo, whereas greater occupancy of the repressor sites is predicted to produce little or no expression.

So how well did the Segal model perform? Using three parameters (concentration scaling, self-cooperativity binding strength and the expression contribution) for each input maternal and gap transcription factor, the model is in general agreement with the gene-expression patterns attributable to the enhancers in the gap genes, but produces only variable agreement with the stripes of gene expression produced by enhancers in the genes known as pair-rule genes, segmentation genes that are expressed in stripes in alternating 'segments' along the anterior-posterior axis. For many of these latter enhancers, there is only a weak correlation between the relative number of activator and repressor binding sites, the nuclear concentrations of the corresponding input transcription factors, and the experimentally observed output gene expression levels. For such enhancers, a more accurate picture means invoking 'nonlinear' mechanisms of transcriptional regulation, such as heterotypic cooperative DNA-binding interactions between different types of activators, repression by quenching (where the binding of a repressor protein appears to prevent activation of the gene by an activator bound at a nearby site) and significant contributions of low-affinity binding sites to the control of gene expression. Indeed, cooperative DNA binding and quenching have proved critical for the accurate modeling of the dorsal-ventral patterning network [10].

Cooperative binding, quenching and the importance of lowaffinity binding sites are well documented in the "presystems' literature. Numerous studies have established the importance of cooperative DNA binding of Bicoid in gene activation [12-14]. Repression by quenching has been shown in the prototypic stripe 2 enhancer that directs the localized expression of the pair-rule gene eve. Although Bicoid-binding activator sites overlap Giant- and Krüppel-binding repressor sites in this enhancer [15,16], both Giant and Krüppel are able to inhibit expression even when bound as much as 50-100 bp away from the closest activator sites $[17,18]$. This quenching is mediated by a co-repressor protein, CtBP, which is recruited to the DNA through interactions with a simple peptide motif, PxDSxK/R, present in the Krüppel, Giant and Knirps gap repressor proteins [19-21]. The eve stripe 2 enhancer contains a total of five Bicoid activator sites, three of which are low-affinity sites [22]. The analysis of natural populations of Drosophila led to the hypothesis that these low-affinity sites provide an intrinsic mechanism for ensuring the robustness of gene expression and enabling the modification of expression patterns during evolution [23,24].

The earlier molecular studies that established these principles were restricted to the analysis of just a handful of enhancers. Segal et al. [2] have now provided evidence that the mechanisms determined previously through traditional molecular studies are prevalent and generally applicable to the entirety of the segmentation process. Indeed, establishing the generality of known mechanisms is one of the great promises of systems biology.

\section{Acknowledgements}

The author thanks Dimitri Papatsenko and Lily Mirels for helpful suggestions. ML is supported by a grant from the NIH (GM3443I).

\section{References}

I. Clyde DE, Corado MS, Wu X, Paré A, Papatsenko D, Small S: A selforganizing system of repressor gradients establishes segmental complexity in Drosophila. Nature 2003, 426:849-853

2. Segal E, Raveh-Sadka T, Schroeder M, Unnerstall U, Gaul U: Predicting expression patterns from regulatory sequence in Drosophila segmentation. Nature 2008, doi:10.1038/nature06496.

3. Berman BP, Nibu Y, Pfeiffer BD, Tomancak P, Celniker SE, Levine M, Rubin GM, Eisen MB: Exploiting transcription factor binding site clustering to identify cis-regulatory modules involved in pattern formation in the Drosophila genome. Proc Natl Acad Sci USA 2002, 99:757-762.

4. Berman BP, Pfeiffer BD, Laverty TR, Salzberg SL, Rubin GM, Eisen $M B$, Celniker SE: Computational identification of developmental enhancers: conservation and function of transcription factor bindingsite clusters in Drosophila melanogaster and Drosophila pseudoobscura. Genome Biol 2004, 5:R6I.

5. Schroeder MD, Pearce M, Fak J, Fan H, Unnerstall U, Emberly E, Rajewsky N, Siggia ED, Gaul U: Transcriptional control in the segmentation gene network of Drosophila. PLoS Biol 2004, 2: 1396-1410.

6. Ochoa-Espinosa A, Yucel G, Kaplan L, Pare A, Pura N, Oberstein A, Papatsenko D, Small S: The role of binding site cluster strength in Bicoid-dependent patterning in Drosophila. Proc Natl Acad Sci USA 2005, 102:4960-4965.

7. Jaeger J, Surkova S, Blagov M, Janssens H, Kosman D, Kozlov KN, Manu, Myasnikova E, Vanario-Alonso CE, Samsonova M, Sharp DH, Reinitz J: Dynamic control of positional information in the early Drosophila embryo. Nature 2004, 430:368-37I.

8. Surkova S, Kosman D, Kozlov K, Manu, Myasnikova E, Samsonova AA, Spirov A, Vanario-Alonso CE, Samsonova M, Reinitz J: Characterization of the Drosophila segment determination morphome. Dev Biol 2008, 313:844-862

9. Bolouri $\mathrm{H}$, Davidson $\mathrm{EH}$ : Transcriptional regulatory cascades in development: initial rates, not steady state, determine network kinetics. Proc Natl Acad Sci USA 2003, 100:937।-9376.

10. Zinzen RP, Senger K, Levine M, Papatsenko D: Computational models for neurogenic gene expression in the Drosophila embryo. Curr Biol 2006, 16:1358-1365.

II. Janssens H, Hou S, Jaeger J, Kim AR, Myasnikova E, Sharp D, Reinitz J: Quantitative and predictive model of transcriptional control of the Drosophila melanogaster even skipped gene. Nat Genet 2006 38: II59-1 I65.

12. Reinitz J, Mjolsness E, Sharp DH: Model for cooperative control of positional information in Drosophila by bicoid and maternal hunchback. J Exp Zool 1995, 271:47-56.

13. Ma X, Yuan D, Diepold K, Scarborough T, Ma J: The Drosophila morphogenetic protein Bicoid binds DNA cooperatively. Development 1996, I22:1 I95-1206.

14. Burz DS, Rivera-Pomar R, Jäckle $H$, Hanes SD: Cooperative DNAbinding by Bicoid provides a mechanism for threshold-dependent gene activation in the Drosophila embryo. EMBO J 1998, 17:5998-6009.

15. Small S, Kraut R, Hoey T, Warrior R, Levine M: Transcriptional regulation of a pair-rule stripe in Drosophila. Genes Dev I99|, 5:827-839.

16. Small S, Blair A, Levine M: Regulation of even-skipped stripe 2 in the Drosophila embryo. EMBO J I992. II:4047-4057.

17. Hewitt GF, Strunk BS, Margulies C, Priputin T, Wang XD, Amey R Pabst BA, Kosman D, Reinitz J, Arnosti DN: Transcriptional repression by the Drosophila giant protein: cis element positioning provides an alternative means of interpreting an effector gradient. Development 1999, 126:1201-1210. 
18. Gray S, Levine M: Short-range transcriptional repressors mediate both quenching and direct repression within complex loci in Drosophila. Genes Dev 1996, 10:700-7I0.

19. Nibu Y, Zhang H, Bajor E, Barolo S, Small S, Levine M: dCtBP mediates transcriptional repression by Knirps, Krüppel and Snail in the Drosophila embryo. EMBO / 1998, 17:7009-7020.

20. Poortinga G, Watanabe M, Parkhurst SM: Drosophila CtBP: a Hairyinteracting protein required for embryonic segmentation and hairymediated transcriptional repression. EMBO J 1998, 17:2067-2078.

21. Nibu Y, Levine MS: CtBP-dependent activities of the short-range Giant repressor in the Drosophila embryo. Proc Natl Acad Sci USA 200I, 98:6204-6208.

22. Arnosti DN, Barolo S, Levine M, Small S: The eve stripe 2 enhancer employs multiple modes of transcriptional synergy. Development 1996, 122:205-2/4.

23. Ludwig MZ, Patel NH, Kreitman M: Functional analysis of eve stripe 2 enhancer evolution in Drosophila: rules governing conservation and change. Development 1998, 125:949-958.

24. Ludwig MZ, Bergman C, Patel NH, Kreitman M: Evidence for stabilizing selection in a eukaryotic enhancer element. Nature 2000, 403:564-567. 